\title{
BOR-FDTD Subgridding Based on Finite Element Principles
}

\author{
Wouter Tierens ${ }^{\mathrm{a}, *}$, Daniël De Zutter ${ }^{\mathrm{a}}$ \\ ${ }^{a}$ Ghent University, Department of Information Technology, Sint-Pietersnieuwstraat 41, \\ Ghent, Belgium
}

\begin{abstract}
In this paper a recently developed provably passive and stable 3D FDTD subgridding technique, based on finite elements principles, is extended to Body-Of-Revolution (BOR) FDTD. First, a suitable choice of basis functions is presented together with the mechanism to assemble them into an overall mesh consisting of coarse and fine mesh cells. Invoking appropriate masslumping concepts then leads to an explicit leapfrog time stepping algorithm for the amplitudes of the basis functions. Attention is devoted to provide the reader with insight into the updating equations, in particular at a subgridding boundary. Stability, grid reflection and dispersion are also discussed. Finally, some numerical examples for toroidal and cylindrical cavities demonstrate the stability and accuracy of the method.
\end{abstract}

Keywords: FDTD methods, BOR-FDTD, Body-Of-Revolution, subgridding, h-refinement

\section{Introduction}

The Finite Difference Time Domain (FDTD) method [1] is one of the most powerful tools in electromagnetic modeling. It is massively parallelizable, matrix-free in contrast to finite-element (FE) techniques, does not require the knowledge of suitable Green's functions, as is the case for integral equation methods and can handle complex geometries. In the past decades a lot of

\footnotetext{
*Corresponding author

Email addresses: Wouter.Tierens@Ugent.be (Wouter Tierens), Daniel.Dezutter@Ugent.be (Daniël De Zutter)
} 
advances have been made with respect to absorbing boundary conditions, dispersion-relation preservation, subgridding, unstructured grids etc. For a review on these advances we refer to $[2,3]$ and the references therein and to the huge body of literature on these topics.

The work presented below was initially motivated by our interest in the complex phenomena that govern the behavior of Tokamak plasmas $[4,5]$ such as to be used in ITER $[6,7]$. When using linearized plasma theory, an FDTD body of revolution (BOR-FDTD) approach can be used, to study each of the independent toroidal modes describing the plasma. In order to be able to predict all relevant wave phenomena, one must be able to correctly model transition regions where the solutions of the dispersion relation can change quite abruptly, involving the sudden and localized appearance of solutions with a wavelength many times shorter than the usual wavelength. This is the so-called mode conversion [5]. As a consequence of the different length scales of these wave phenomena, subgridding is indicated to alleviate the computational cost of maintaining an identical cell size over the complete problem space.

In this paper we report on our efforts to develop a suitable BOR-FDTD subgridding technique which can of course be used outside the context of plasma research. For an introduction to BOR-FDTD we refer to Chapter 12 of [1] and to e.g., [8, 9, 10, 11, 12]. Subgridding has been thoroughly investigated in the past $[13,14,15,16,17,18,19,20,21,22,23]$. To develop BOR-FDTD subgridding, our starting point is the work of Chilton et. al. [17, 24] in which conservative, provably stable and explicit 3D FDTD subgridding methods are developed based on finite-element concepts.

This paper is organised as follows. Following the ideas put forward in [17, 24], Section 2 presents a new set of basis functions particularly tailored for BOR-FDTD, whereby electric field and magnetic induction basis functions explicitly satisfy Faraday's law. It is shown that, under certain assumptions, the classical BOR-FDTD update equations for a uniform grid [1] can be recovered. Section 3 introduces subgridding while Section 4 explains how to assemble a complete grid consisting of coarse and fine meshes. In Section 5 particular attention is devoted to the mass-lumping concepts that have to be introduced in order to obtain a provably stable and explicit method. Section 6 then presents the final leapfrog time stepping algorithm, taking care to provide insight on how the complete mathematical machinery leads to updating equations at the interfaces between coarse and fine meshes, update equations that are no longer intuitive. Section 7 presents some numerical 


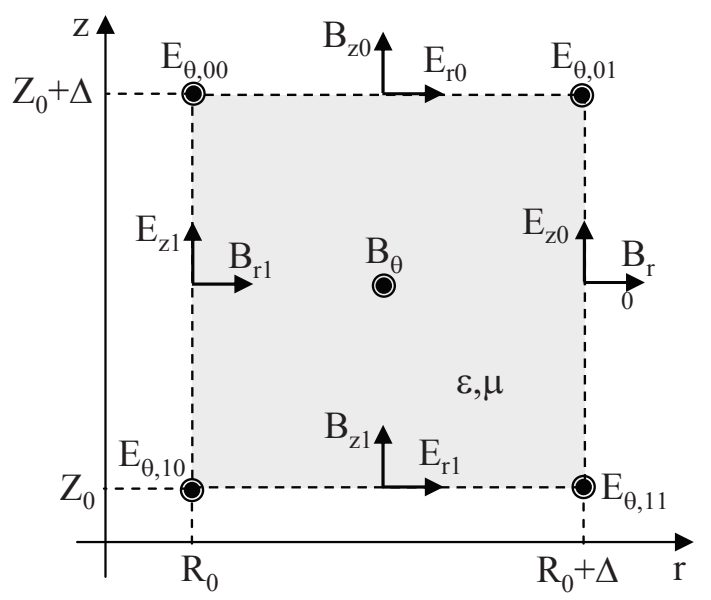

Figure 1: A Yee-like unit cell in BOR-FDTD showing the anchor points of the basis functions.

results. First, stability, grid reflection and grid dispersion are discussed. Next, the field analysis in a toroidal perfectly conducting (PEC) cavity is used to illustrate the stability and accuracy of the subgridding. Finally, the resonance frequency of a reentrant cylindrical cavity as encountered in Alvarez-type particle accelerators, is calculated, showing that subgridding at the reentrant corner can substantially reduce simulation time and memory resources while retaining accuracy. The final section formulates a number of conclusions and future challenges.

\section{BOR-FDTD discretisation of Maxwell's equations}

\subsection{Basis functions}

As our starting point we take the classical BOR-FDTD representation of [1]. Fig. 1 shows a unit BOR-FDTD cell with material parameters $\epsilon$ and $\mu$ centered on the $B_{\theta}$ component, extending from $r=R_{0}$ to $r=R_{0}+\Delta$ and from $z=Z_{0}$ to $z=Z_{0}+\Delta$. For each angular mode $M$, the following 8 basis 
functions $\vec{E}_{n}$ for the electric field are introduced:

$$
\begin{aligned}
& E_{r i}=\left(\left\{\begin{array}{ll}
\frac{z-Z_{0}}{\Delta} & i=0 \\
1-\frac{z-Z_{0}}{\Delta} & i=1
\end{array}\right) \cos (M \theta) \overrightarrow{1}_{r}\right. \\
& E_{\theta, i j}=\left(\{ \begin{array} { l l } 
{ \frac { z - Z _ { 0 } } { \Delta } } & { i = 0 } \\
{ 1 - \frac { z - Z _ { 0 } } { \Delta } } & { i = 1 }
\end{array} ) \cdot \left(\left\{\begin{array}{ll}
f_{1}(r) & j=0 \\
f_{2}(r) & j=1
\end{array}\right) \sin (M \theta) \overrightarrow{1}_{\theta}\right.\right. \\
& E_{z i}=\left(\left\{\begin{array}{ll}
\frac{r-R_{0}}{\Delta} & i=0 \\
1-\frac{r-R_{0}}{\Delta} & i=1
\end{array}\right) \cos (M \theta) \overrightarrow{1}_{z}\right.
\end{aligned}
$$

with $f_{1}(r)=\frac{R_{0}}{\Delta}\left(\frac{R_{0}+\Delta}{r}-1\right)$ and $f_{2}(r)=\frac{R_{0}+\Delta}{\Delta}\left(1-\frac{R_{0}}{r}\right)$. These basis functions are zero outside the considered cell. Their anchor points are also shown in Fig. 1. Each basis function is equal to one in its anchor point and zero in the other anchor points of corresponding field components, e.g. $E_{\theta, 00}$ is equal to one in the upper left corner and zero in the three other corners. In BOR-FDTD calculations, the counterparts of (1)-(3) with $\cos (M \theta)$ replaced by $\sin (M \theta)$ and $\sin (M \theta)$ by $-\cos (M \theta)$ are also needed but will not be further discussed. At this point it should be remarked that the above defined basis functions are not curl conforming, but the grid assembling procedure described in Section 4 will result in the fact that the tangential component of the electric field becomes continuous everywhere. To be able to apply the procedures outlined in [24], the 5 basis functions $\overrightarrow{B_{n}}$ for the magnetic induction must satisfy the curl inclusion property: the curl of a linear combination of electric field basis functions must be a linear combination of magnetic induction basis functions. This is the case for (again see Fig. 1)

$$
\begin{aligned}
B_{r i} & =\frac{\left(R_{0}+(1-i) \Delta\right) \sin (M \theta)}{r} \cdot\left(\left\{\begin{array}{ll}
\frac{r-R_{0}}{\Delta} & i=0 \\
1-\frac{r-R_{0}}{\Delta} & i=1
\end{array}\right) \overrightarrow{1}_{r}\right. \\
B_{\theta} & =\cos (M \theta) \overrightarrow{1}_{\theta} \\
B_{z i} & =\frac{\left(R_{0}+\Delta / 2\right) \sin (M \theta)}{r} \cdot\left(\left\{\begin{array}{ll}
\frac{z-Z_{0}}{\Delta} & i=0 \\
1-\frac{z-Z_{0}}{\Delta} & i=1
\end{array}\right) \overrightarrow{1}_{z}\right.
\end{aligned}
$$

Note that for $R_{0} \gg \Delta$ all basis functions reduce to linear functions in $\alpha=$ $\left(r-R_{0}\right) / \Delta$ and $z$, recovering the cartesian limit. Next, Faraday's law $\vec{\nabla} \times \vec{E}=$ $-\frac{\partial \vec{B}}{\partial t}$ is discretized using the above defined basis functions, leading to its discrete counterpart

$$
C \mathbf{e}(t)=-\frac{d \mathbf{b}(t)}{d t}
$$


with e the column vector $\left[e_{r 0}, e_{r 1}, e_{\theta, 00}, e_{\theta, 01}, e_{\theta, 10}, e_{\theta, 11}, e_{z 0}, e_{z 1}\right]^{T}$ of electric field basis function amplitudes, $\mathbf{b}=\left[b_{r 0}, b_{r 1}, b_{\theta}, b_{z 0}, b_{z 1}\right]^{T}$ the corresponding magnetic induction basis function amplitudes and with $C^{T}$ given by

$$
\left[\begin{array}{ccccc}
0 & 0 & \frac{1}{\Delta} & \frac{M}{R_{0}+\Delta / 2} & 0 \\
0 & 0 & \frac{-1}{\Delta} & 0 & \frac{M}{R_{0}+\Delta / 2} \\
0 & \frac{-1}{\Delta} & 0 & \frac{-R_{0}}{\Delta\left(R_{0}+\Delta / 2\right)} & 0 \\
\frac{-1}{\Delta} & 0 & 0 & \frac{R_{0}+\Delta}{\Delta\left(R_{0}+\Delta / 2\right)} & 0 \\
0 & \frac{1}{\Delta} & 0 & 0 & \frac{-R_{0}}{\Delta\left(R_{0}+\Delta / 2\right)} \\
\frac{1}{\Delta} & 0 & 0 & 0 & \frac{R_{0}+\Delta}{\Delta\left(R_{0}+\Delta / 2\right)} \\
\frac{-M}{R_{0}+\Delta} & 0 & \frac{-1}{\Delta} & 0 & 0 \\
0 & \frac{-M}{R_{0}} & \frac{1}{\Delta} & 0 & 0
\end{array}\right]
$$

Ampere's law $\vec{\nabla} \times \mu^{-1} \vec{B}=\epsilon \frac{\partial \vec{E}}{\partial t}$ is satisfied weakly by testing both sides with the electric field basis functions. This finally leads to

$$
\left[\star_{\epsilon}\right]^{-1} C^{T}\left[\star_{\mu}^{-1}\right] \mathbf{b}(t)=\frac{d \mathbf{e}(t)}{d t}
$$

where $\left[\star_{\mu}^{-1}\right]$ and $\left[\star_{\epsilon}\right]$ are the mass matrices obtained by integrating scalar products of basis functions over the whole problem volume.

$$
\begin{aligned}
{\left[\star_{\mu}^{-1}\right]_{n, m} } & =\int \mu^{-1} \overrightarrow{B_{n}} \cdot \overrightarrow{B_{m}} d V \\
{\left[\star_{\epsilon}\right]_{n, m} } & =\int \epsilon \overrightarrow{E_{n}} \cdot \overrightarrow{E_{m}} d V
\end{aligned}
$$

The integration over $\theta$ is performed analytically, yielding a factor of $\pi$. The remaining integration over $r$ and $z$ could also be performed analytically. However, approximating these integrals using second-order accurate trapezoidal integration for both the integration over $r$ and over $z$ - socalled mass lumping [25] - makes these matrices diagonal with elements $\left[\star_{\epsilon}\right]_{n, n}=\frac{\epsilon \pi \Delta^{2} R_{0}}{4}\left(2+\Delta^{\prime}, 2+\Delta^{\prime}, 1,1+\Delta^{\prime}, 1,1+\Delta^{\prime}, 2+2 \Delta^{\prime}, 2\right),\left[\star_{\mu}^{-1}\right]_{n, n}=$ $\frac{\mu^{-1} \pi \Delta^{2} R_{0}}{2}\left(1+\Delta^{\prime}, 1,2+\Delta^{\prime}, 1+0.5 \Delta^{\prime}, 1+0.5 \Delta^{\prime}\right)$ and $\Delta^{\prime}=\Delta / R_{0}$. The 8 electric field basis functions were ordered as follows: $E_{r 0}, E_{r 1}, E_{\theta, 00}, E_{\theta, 01}$, $E_{\theta, 10}, E_{\theta, 11}, E_{z 0}, E_{z 1}$ and the 5 magnetic basis functions as $B_{r 0}, B_{r 1}, B_{\theta}$, $B_{z 0}, B_{z 1}$. The above mentioned anchor point property of the basis functions is a necessary condition for the trapezoidal integration to lead to a diagonal 
matrix. The reader also notices that each entry of the mass matrices is proportional to the $r$-coordinate of the anchor point of the corresponding basis function. However, when performing the calculations for the $B_{z}$ components, the reader will find out that this is only the case provided terms of the order of $O\left(\Delta^{4}\right)$ are neglected, totally in line with the fact that the trapezoidal integration is only second order accurate in $\Delta$. Although this proportionality with the radius of the anchor point is not strictly required, it turns out that in this way the mass-lumped finite element approach that we are using here, exactly recovers the classical BOR-FDTD update equations.

\section{Subgridding basis functions}

Starting from the above discretisation of Maxwell's equations for basis functions defined on a $\Delta$ by $\Delta$ grid, our aim is to obtain a conservative and stable subgridding procedure. Suppose that subgrids of size $\Delta / N \times \Delta / N$, with $N$ an integer, are introduced. In order to be able to apply the theory put forward in [24], asserting that the resulting scheme is indeed conservative and stable, the basis functions on the $\Delta / N \times \Delta / N$ grid much be such that a linear combination of them yields the basis functions of the $\Delta \times \Delta$ grid. With this in mind, et us take a look at the four $E_{\theta, i j}$ functions defined in (2). In eq. 12 they are be cast in a matrix form. The r.h.s. coefficient matrix is non-singular and hence invertible. Consequently, 1 (the constant), $z, z / r$ and $1 / r$ (and all linear combinations thereof) can be written as linear combinations of the four $E_{\theta}$ basis functions.

$$
\begin{aligned}
& {\left[\begin{array}{llll}
E_{\theta, 00} & E_{\theta, 01} & E_{\theta, 10} & E_{\theta, 11}
\end{array}\right]^{T}=\frac{\sin (M \theta)}{\Delta^{2}}} \\
& {\left[\begin{array}{cccc}
0 & -R_{0} & R_{0}\left(R_{0}+\Delta\right) & 0 \\
0 & R_{0}+\Delta & -R_{0}\left(R_{0}+\Delta\right) & 0 \\
-\Delta R_{0} & R_{0} & -R_{0}\left(R_{0}+\Delta\right) & \Delta R_{0}\left(R_{0}+\Delta\right) \\
\Delta\left(R_{0}+\Delta\right) & -\left(R_{0}+\Delta\right) & R_{0}\left(R_{0}+\Delta\right) & -\Delta R_{0}\left(R_{0}+\Delta\right)
\end{array}\right]\left[\begin{array}{c}
1 \\
z-Z_{0} \\
\frac{z-Z_{0}}{r} \\
\frac{1}{r}
\end{array}\right]}
\end{aligned}
$$

We now define the $E_{\theta}$ subgrid basis functions on the $\Delta / N \times \Delta / N$ grid in the same way as in (2), replacing $\Delta$ by $\Delta / N$ for a subgrid cell extending from $r=R_{0}$ to $r=R_{0}+\Delta / N$ and from $z=Z_{0}$ to $z=Z_{0}+\Delta / N$ (and similarly if the origin of the cell is located elsewhere). It then follows that the $E_{\theta}$ basis functions on a coarse cell, being linear combinations of $1, z, z / r$ and 
$1 / r$, can be written as linear combinations of the $E_{\theta}$ functions on the finer cells nested in the coarse cell. The same reasoning applies to the $E_{r}$ basis functions which form a basis for all linear combinations of 1 and $z$, the $E_{z}$ basis functions for 1 and $r$, the $B_{r}$ basis functions for $1 / r$ and 1 , the $B_{z}$ basis functions for $1 / r$ and $z / r$ and the $B_{\theta}$ functions for 1 .

\section{Grid assembling}

Following [24], we now proceed to assemble cells into a complete mesh. The starting point is a coarse and a fine mesh that are still unconnected. Next, restriction operators that combine field quantities at each subgridding edge are introduced. On the edges of the fine grid, only field quantities of the coarse grid are retained, as will become clear from a small example below. However, in order to guarantee stability, the restriction operators must obey certain commutation properties. Let $A_{E}$ be the restriction operator for the electric fields and $A_{B}$ for the magnetic inductions. Using these operators, the Maxwell curl equations for the complete mesh become

$$
\begin{gathered}
C_{\text {joint }} \mathbf{e}(t)=-\frac{d \mathbf{b}(t)}{d t} \\
{\left[\star_{\epsilon}\right]^{-1} C_{\text {joint }}^{T}\left[\star_{\mu}^{-1}\right] \mathbf{b}(t)=\frac{d \mathbf{e}(t)}{d t}} \\
{\left[\star_{\epsilon}\right]=A_{E}\left[\star_{\epsilon}\right]_{\text {disjoint }} A_{E}^{T}} \\
{\left[\star_{\mu}^{-1}\right]=A_{B}\left[\star_{\mu}^{-1}\right]_{\text {disjoint }} A_{B}^{T}} \\
C_{\text {joint }}=\left(A_{B} A_{B}^{T}\right)^{-1} A_{B} C_{\text {disjoint }} A_{E}^{T}
\end{gathered}
$$

with $A_{E} C_{\text {disjoint }}^{T}=C_{\text {joint }}^{T} A_{B}$. The subscript disjoint indicates that the corresponding curl and mass matrices are those as derived in previous sections. Suppose the original disjoint grids have $N_{B, c}$ and $N_{B, f}$ magnetic field discretization points and $N_{E, c}$ and $N_{E, f}$ electric field discretization points. Subscripts $c$ and $f$ indicate coarse resp. fine grid cells. $N_{E, \cap}$ of the fine electric field discretization points and $N_{B, \cap}$ of the fine magnetic field discretization points are on the subgridding edge. For a rectangular subgrid, $A_{E}$ is a $\left(N_{E, c}+N_{E, f}-N_{E, \cap}\right) \times\left(N_{E, c}+N_{E, f}\right)$ matrix and $A_{B}$ is a $\left(N_{B, c}+N_{B, f}-N_{B, \cap}\right) \times\left(N_{B, c}+N_{B, f}\right)$ matrix.

To clarify the above concepts, the reader can of course consult [24], but as this paper deals with the BOR-FDTD case with its very specific arrangement of basis functions, let us take a look at a typical restriction process. 
We first consider the case in which a complete mesh consisting of identical cells is assembled, i.e. only coarse cells are present. Let us concentrate on the magnetic induction, the electric fields can be treated analogously. Fig. 2 shows a simple example consisting of two cells. To the left (Fig. 2a) the cells are shown separately and the positions of the B-field basis functions are indicated by the black dots. To the right (Fig. 2b) the cells have been assembled into "the mesh" and a global numbering is introduced. For this example we have that $N_{B, c}=2 \times 5$ and $N_{B, \cap}=1$. Collecting all the original magnetic induction basis functions in the $10 \times 1$ column vector $\mathbf{b}_{\text {disjoint }}=\left[b_{1}, \ldots, b_{10}\right]^{T}$ and the ones pertaining to the mesh of Fig. $2 \mathrm{~b}$ in the $9 \times 1$ column vector $\mathbf{b}_{\text {joint }}=\left[b_{1}, \ldots, b_{9}\right]^{T}$, we have that $\mathbf{b}_{\text {joint }}=A_{B} \mathbf{b}_{\text {disjoint }}$ with $A_{B}$ the $9 \times 10$ magnetic induction restriction matrix. The non-zero elements of this matrix are given by: $A_{B, 1,1}=A_{B, 1,8}=A_{B, 2,2}=A_{B, 3,3}=A_{B, 4,4}=A_{B, 5,5}=A_{B, 6,6}=$ $A_{B, 7,7}=A_{B, 8,9}=A_{B, 9,10}=1$. Note that all non-zero values are identical and equal to one. Of course, adding more cells will lead to additional couplings. Here only a coupling in a single point occurs. For the electric fields the same example leads to three coupled basis functions and with an analogous notation as above we have that $\mathbf{e}_{\text {joint }}=A_{E} \mathbf{e}_{\text {disjoint }}$ with $\mathbf{e}_{\text {disjoint }}$ a $16 \times 1$ column vector, $\mathbf{e}_{\text {joint }}$ a $13 \times 1$ column vector and $A_{E}$ the $13 \times 16$ electric field restriction matrix (with 16 non-zero entries equal to one).

Let us now turn to a subgridding example. Fig. 3a shows a single coarse cell and 3 fine mesh cells $(N=3)$ before fusing them together. Fig. 3b displays the resulting connected mesh (see also Fig. 4 ). Note that in the connected mesh only coarse mesh field values are used at the edge connecting the coarse and the fine mesh. For this subgridding example we now have that $N_{B, c}=5, N_{B, f}=3 \times 5$ and $N_{B, \cap}=3+1+1$, i.e. the 3 fine mesh points labeled 8,13 and 18 and 2 points to connect the fine mesh cells between them, the points labeled 7 and 12. For points 1, 8, 13 and 18 the corresponding expansion functions of interest, leaving out the $\sin (M \theta)$ factor, are:

$$
\begin{gathered}
B_{z, c}=\frac{\left(R_{0}+\Delta / 2\right)}{r}\left(1-\frac{z-Z_{0}}{\Delta}\right) \\
B_{z, f i}=\frac{\left(R_{0}+(i-1 / 2) \Delta / 3\right) \sin (M \theta)}{r}\left(1+3 \frac{z-Z_{0}}{\Delta}\right)
\end{gathered}
$$

with $i=1,2,3$, for resp. points 8,13 and 18 . From the above it is clear that the coarse cell basis function at $z=Z_{0}$, i.e. at the subgridding edge, can be 


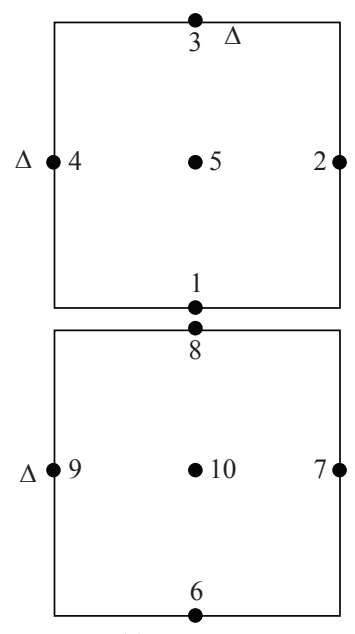

(a)

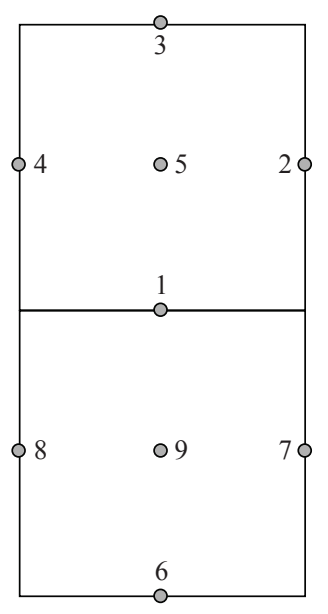

(b)

Figure 2: (a) two cells with anchor points and numbering for the magnetic induction basis functions. (b) assembled cells with global numbering.

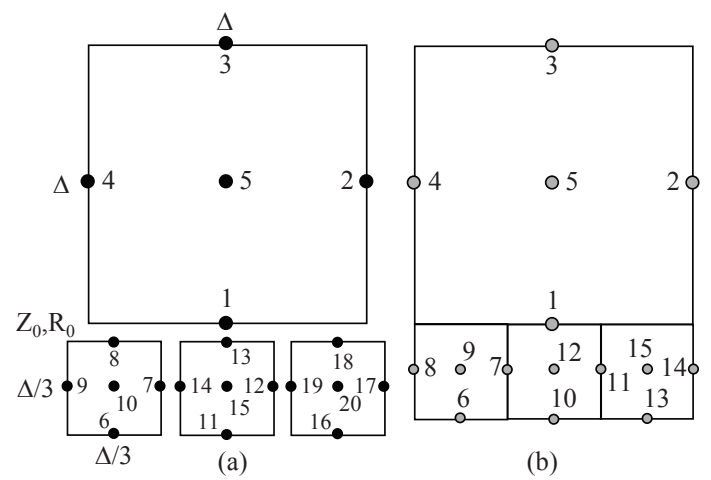

Figure 3: (a) one coarse mesh cell and 3 fine mesh cells with numbering for the magnetic induction basis functions. (b) assembled cells with global numbering. 

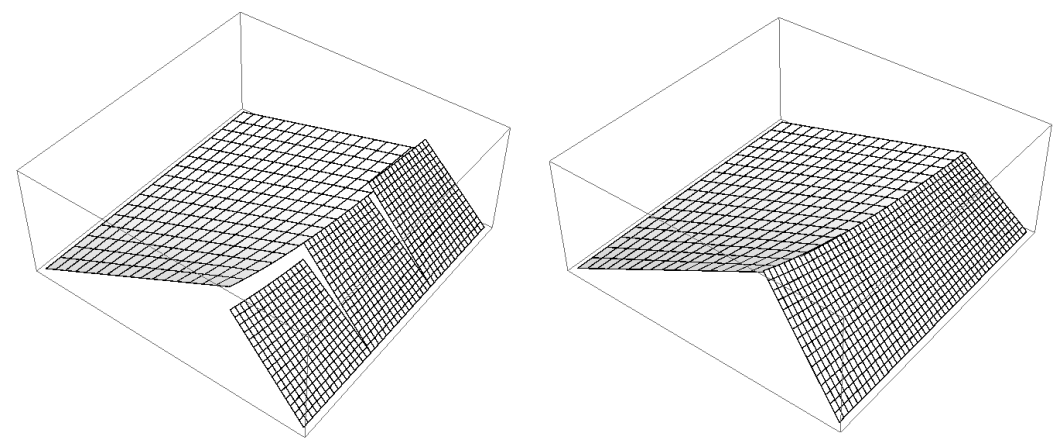

Figure 4: Disjoint $\vec{B}_{z}$ basis functions combine to form a single joint basis function that is continuous at the cell boundary.

written as the following linear combination of fine cell functions:

$$
\begin{aligned}
& \left.B_{z, c}\right|_{z=Z_{0}}= \\
& \left.\left(\frac{R_{0}+\Delta / 2}{R_{0}+\Delta / 6} B_{z, f 1}+B_{z, f 2}+\frac{R_{0}+\Delta / 2}{R_{0}+5 \Delta / 6} B_{z, f 3}\right)\right|_{z=Z_{0}}
\end{aligned}
$$

This is a simply illustration of the general properties announced in Section 3. Note that (18) guarantees pointwise $B_{z}$ flux continuity along the subgridding edge. In this subgridding example the $15 \times 20$ magnetic induction restriction matrix $A_{B}$ again satisfies $\mathbf{b}_{\text {joint }}=A_{B} \mathbf{b}_{\text {disjoint }}$, with $\mathbf{b}_{\text {joint }}$ resp. $\mathbf{b}_{\text {disjoint }}$ a $15 \times 1$ resp. $20 \times 1$ column vector. The 20 non-zero elements of $A_{B}$ for this case are given by: $A_{B, 1,1}=A_{B, 2,2}=A_{B, 3,3}=A_{B, 4,4}=A_{B, 5,5}=A_{B, 6,6}=$ $A_{B, 7,7}=A_{B, 7,14}=A_{B, 8,9}=A_{B, 9,10}=A_{B, 10,11}=A_{B, 11,12}=A_{B, 11,19}=$ $A_{B, 12,15}=A_{B, 13,16}=A_{B, 14,17}=A_{B, 15,20}=1, A_{B, 1,8}=\frac{R_{0}+\Delta / 2}{R_{0}+\Delta / 6}, A_{B, 1,13}=1$ and $A_{B, 1,18}=\frac{R_{0}+\Delta / 2}{R_{0}+5 \Delta / 6}$. The last three elements correspond to the basis functions coefficients in the r.h.s. of (18) or, put differently, the first row of $A_{B}$ enforces identity (18).

\section{Mass lumping}

By now, the grid assembly process should be clear. However, before turning to the time-stepping part of the algorithm, special attention has to be devoted to the mass matrices $\left[\star_{\epsilon}\right]$ and $\left[\star_{\mu}^{-1}\right]$ in (14). Although the original mass matrices for a single BOR-FDTD cell are diagonal (see the end 


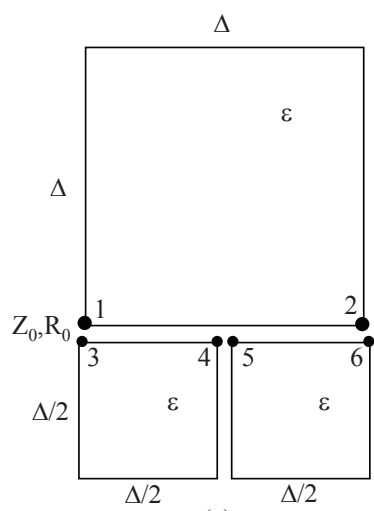

(a)

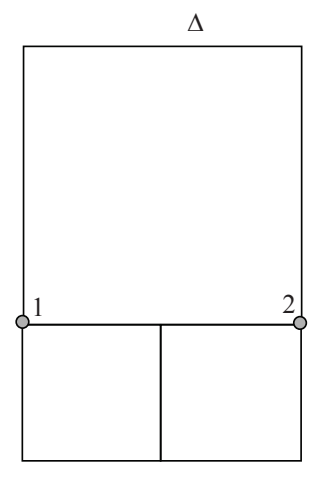

(b)

Figure 5: Relevant to mass lumping for $\vec{E}_{\theta}$ : (a) one coarse mesh cell and 2 fine mesh cells with anchor points and numbering for the $\vec{E}_{\theta}$ basis functions; (b) assembled cells with global numbering.

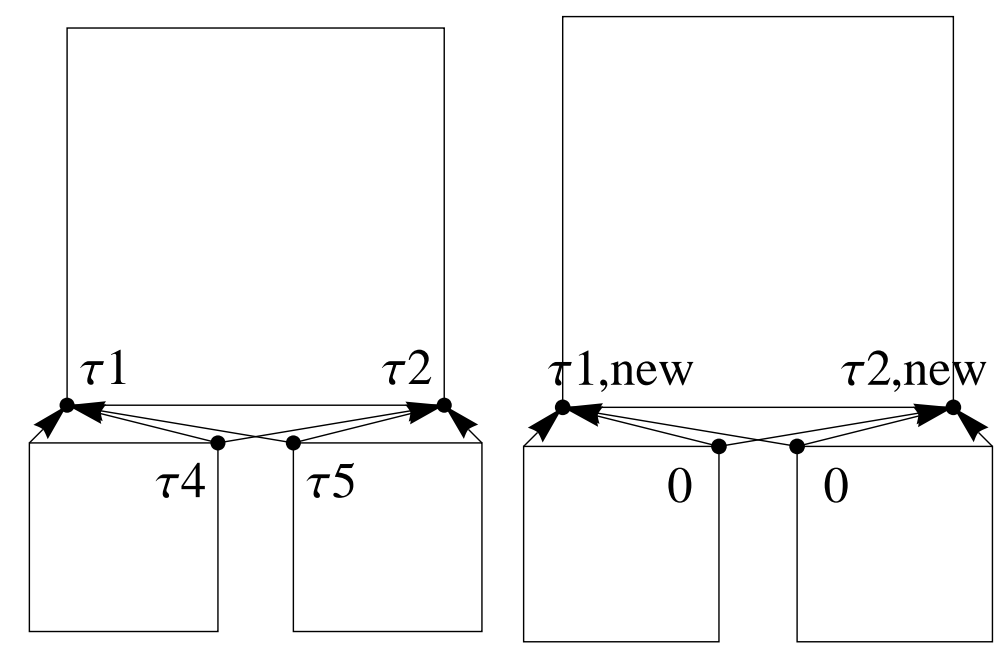

Figure 6: The "mass" associated with the fine basis functions that couple to multiple coarse basis functions is redistributed. 
of Section 2) the assembly operators can reintroduce non-diagonal elements and here again some type of mass lumping will have to be introduced if we want to preserve the diagonal character and hence the explicit timestepping properties. Let us again look at an elucidating example in which the assembly process leads to a non-diagonal $\left[\star_{\epsilon}\right]$ matrix. Fig. 5 shows a single coarse cell and, for the sake of simplicity, two fine mesh cells, before and after fusing them together. We concentrate on the $E_{\theta}$ field components on the subgridding boundary, six in total in the original configuration with two of them (the coarse grid points) remaining after assembling the coarse cell with the two fine ones. The relevant expansion functions are given by:

$$
\begin{array}{cc}
E_{\theta, 1 c}=Z_{c}(z) \frac{R_{0}}{\Delta}\left(\frac{R_{0}+\Delta}{r}-1\right) & E_{\theta, 2 c}=Z_{c}(z) \frac{R_{0}+\Delta}{\Delta}\left(1-\frac{R_{0}}{r}\right) \\
E_{\theta, 3 f}=Z_{f}(z) \frac{2 R_{0}}{\Delta}\left(\frac{R_{0}+\Delta / 2}{r}-1\right) & E_{\theta, 4 f}=Z_{f}(z) \frac{R_{0}+\Delta / 2}{\Delta / 2}\left(1-\frac{R_{0}}{r}\right) \\
E_{\theta, 5 f}=Z_{f}(z) \frac{R_{0}+\Delta / 2}{\Delta / 2}\left(\frac{R_{0}+\Delta}{r}-1\right) & E_{\theta, 6 f}=Z_{f}(z) \frac{R_{0}+\Delta}{\Delta / 2}\left(1-\frac{R_{0}+\Delta / 2}{r}\right) \\
Z_{c}(z)=\left(1-\frac{z-Z_{0}}{\Delta}\right) & Z_{f}(z)=\left(1+2 \frac{z-Z_{0}}{\Delta}\right)
\end{array}
$$

The pertinent linear combination of fine cell basis functions, to obtain the corresponding coarse ones, is:

$$
\begin{gathered}
\left.E_{\theta, 1 c}\right|_{z=Z_{0}}=E_{\theta, 3 f}+\left.\alpha\left(E_{\theta, 4 f}+E_{\theta, 5 f}\right)\right|_{z=Z_{0}} \\
\left.E_{\theta, 2 c}\right|_{z=Z_{0}}=E_{\theta, 6 f}+\left.\beta\left(E_{\theta, 4 f}+E_{\theta, 5 f}\right)\right|_{z=Z_{0}} \\
\alpha=\frac{R_{0}}{2\left(R_{0}+\Delta / 2\right)} \\
\beta=\frac{R_{0}+\Delta}{2\left(R_{0}+\Delta / 2\right)}
\end{gathered}
$$

Only considering the six basis functions in the example, the $2 \times 6$ electric field restriction matrix $A_{E}$ has 8 non-zero entries: $A_{E, 1,1}=A_{E, 1,3}=A_{E, 2,2}=$ $A_{E, 2,6}=1, A_{E, 1,4}=A_{E, 1,5}=\alpha$ and $A_{E, 2,4}=A_{E, 2,5}=\beta$, while the diagonal entries of the disjoint electric field mass matrix $\left[\star_{\epsilon}\right]_{\text {disjoint }}$ are:

$$
\begin{aligned}
& \left(\tau_{1}, \tau_{2}, \tau_{3}, \tau_{4}, \tau_{5}, \tau_{6}\right)= \\
& \quad \frac{\epsilon \pi \Delta^{2} R_{0}}{16}\left(4,4\left(1+\Delta^{\prime}\right), 1,1+\Delta^{\prime} / 2,1+\Delta^{\prime} / 2,1+\Delta^{\prime}\right)
\end{aligned}
$$

We have, for simplicity, assumed that the value of $\epsilon$ does not change from one cell to the other. All this finally implies that the corresponding electric 
field $2 \times 2$ mass matrix $\left[\star_{\epsilon}\right]$ of the joined cells has the following elements:

$$
\begin{gathered}
\epsilon_{11}=\tau_{1}+\tau_{3}+\alpha^{2}\left(\tau_{4}+\tau_{5}\right) \\
\epsilon_{12}=\epsilon_{21}=\alpha \beta\left(\tau_{4}+\tau_{5}\right) \\
\epsilon_{22}=\tau_{2}+\tau_{6}+\beta^{2}\left(\tau_{4}+\tau_{5}\right)
\end{gathered}
$$

showing that the grid assembling process can give rise to non-diagonal mass matrices.

To prevent the restriction operator from introducing these non-diagonal elements the following procedure has to be adopted:

1) Identify the disjoint basis functions which couple to multiple joint basis functions. In the example of Fig. 5 these disjoint basis functions are those associated with anchor point 4 and 5 . In general this amounts to identifying columns in the restriction matrix with more than one non-zero element.

2) Set the corresponding elements on the diagonal of $\left[\star_{\epsilon}\right]_{\text {disjoint }}$ to zero. In our example this implies that $\tau_{4}$ and $\tau_{5}$ must be set to zero.

3) For each of the considered disjoint basis functions, redistribute the original value on the diagonal over the multiple joint basis functions it couples with. The relevant joint basis functions are easily identified as they correspond to the row indices of the non-zero elements in the corresponding disjoint basis function column. In our example disjoint basis function 4 couples with joint basis functions 1 and 2 and the same is the case for disjoint basis function 5. In BOR-FDTD the number of joint basis functions that couple to multiple disjoint ones is at most two.

4) The redistribution over the two joint basis functions is carried out in such a way that the value added to $\left[\star_{\epsilon}\right]_{\text {disjoint,jj }}(j=1,2)$, is proportional to $\left[\star_{\epsilon}\right]_{\text {disjoint }, j j}$ and that the trace of the $\left[\star_{\epsilon}\right]_{\text {disjoint }}$ matrix remains unaltered. The example of Fig. 5, in which a redistribution over two basis functions has to be carried out, will clarify the procedure. Both $\tau_{4}$ and $\tau_{5}$ have to be redistributed over $\tau_{1}$ and $\tau_{2}$ as follows:

$$
\begin{gathered}
\tau_{1, \text { new }}=\tau_{1}+\frac{\tau_{1}}{\tau_{1}+\tau_{2}} \tau_{4}+\frac{\tau_{1}}{\tau_{1}+\tau_{2}} \tau_{5}=\tau_{1}+\alpha\left(\tau_{4}+\tau_{5}\right) \\
\tau_{2, \text { new }}=\tau_{2}+\frac{\tau_{2}}{\tau_{1}+\tau_{2}} \tau_{4}+\frac{\tau_{2}}{\tau_{1}+\tau_{2}} \tau_{5}=\tau_{2}+(1-\alpha)\left(\tau_{4}+\tau_{5}\right)
\end{gathered}
$$

with $\alpha=\frac{\tau_{1}}{\tau_{1}+\tau_{2}}$. This redistribution is depicted in Fig. 6. Using these new values $\tau_{1, \text { new }}$ and $\tau_{2, \text { new }}$, with $\tau_{4, \text { new }}=\tau_{5, \text { new }}=0$, the diagonal entries of 
$\left[\star_{\epsilon}\right]_{\text {disjoint,new }}$ become:

$$
\begin{array}{r}
\left(\tau_{1, \text { new }}, \tau_{2, \text { new }}, \tau_{3, \text { new }}, \tau_{4, \text { new }}, \tau_{5, \text { new }}, \tau_{6, \text { new }}\right)= \\
\frac{\epsilon \pi \Delta^{2} R_{0}}{16}\left(5,5\left(1+\Delta^{\prime}\right), 1,0,0,1+\Delta^{\prime}\right)
\end{array}
$$

It is easily verified that the total sum remains unaltered and that $\frac{\tau_{1}}{\tau_{2}}=\frac{\tau_{1, \text { new }}}{\tau_{2, \text { new }}}$.

5) With this new disjoint mass matrix, the new joint mass matrix can be determined. By construction, this matrix will now be diagonal. In our example we have

$$
\begin{gathered}
\epsilon_{11, \text { new }}=\tau_{1, \text { new }}+\tau_{3}=\frac{3 \epsilon \pi \Delta^{2} R_{0}}{8} \\
\epsilon_{12, \text { new }}=\epsilon_{21, \text { new }}=0 \\
\epsilon_{22, \text { new }}=\tau_{2, \text { new }}+\tau_{6}=\frac{3 \epsilon \pi \Delta^{2} R_{0}}{8}\left(1+\Delta^{\prime}\right)
\end{gathered}
$$

It should be noted that in BOR-FDTD only the $E_{\theta}$ components can give rise to a non-diagonal $\left[\star_{\epsilon}\right]$ matrix and to which the above procedure must be applied.

\section{Leapfrog time stepping}

The final BOR-FDTD equations are given by (12) and (13). Timestepping of these equations is straightforward. It suffices to e.g. discretise $\mathbf{b}$ in (12) on whole time steps $n \Delta t$ and $\mathbf{e}$ on half time steps $(n+1 / 2) \Delta t$ to obtain the following leapfrog updating scheme:

$$
\begin{gathered}
\mathbf{b}_{n}=\mathbf{b}_{n-1}-C_{\text {joint }} \mathbf{e}_{n-1 / 2} \Delta t \\
\mathbf{e}_{n+1 / 2}=\mathbf{e}_{n-1 / 2}+\left[\star_{\epsilon}\right]^{-1} C_{\text {joint }}^{T}\left[\star_{\mu}^{-1}\right] \mathbf{b}_{n} \Delta t
\end{gathered}
$$

This can be cast into the following form

$$
\begin{aligned}
& {\left[\begin{array}{ll}
\tilde{\mathbf{e}}_{n+1 / 2} & \tilde{\mathbf{b}}_{n}
\end{array}\right]^{T}=} \\
& {\left[\begin{array}{cc}
\mathbf{I}-\tilde{C}_{\text {joint }}^{T} \tilde{C}_{\text {joint }}(\Delta t)^{2} & \tilde{C}_{\text {joint }}^{T} \Delta t \\
-\tilde{C}_{\text {joint }} \Delta t & \mathbf{I}
\end{array}\right]\left[\begin{array}{c}
\tilde{\mathbf{e}}_{n-1 / 2} \\
\tilde{\mathbf{b}}_{n-1}
\end{array}\right]}
\end{aligned}
$$

or $\mathbf{u}_{n}=\mathbf{A} \mathbf{u}_{n-1}$, with $\mathbf{A}$ the so-called amplification matrix, $\tilde{\mathbf{e}}=\left[\star_{\epsilon}\right]^{1 / 2} \mathbf{e}$, $\tilde{\mathbf{b}}=\left[\star_{\mu}^{-1}\right]^{1 / 2} \mathbf{b}$ and $\tilde{C}_{\text {joint }}=\left[\star_{\mu}^{-1}\right]^{1 / 2} C_{\text {joint }}\left[\star_{\epsilon}\right]^{-1 / 2}$. The reader is referred to 


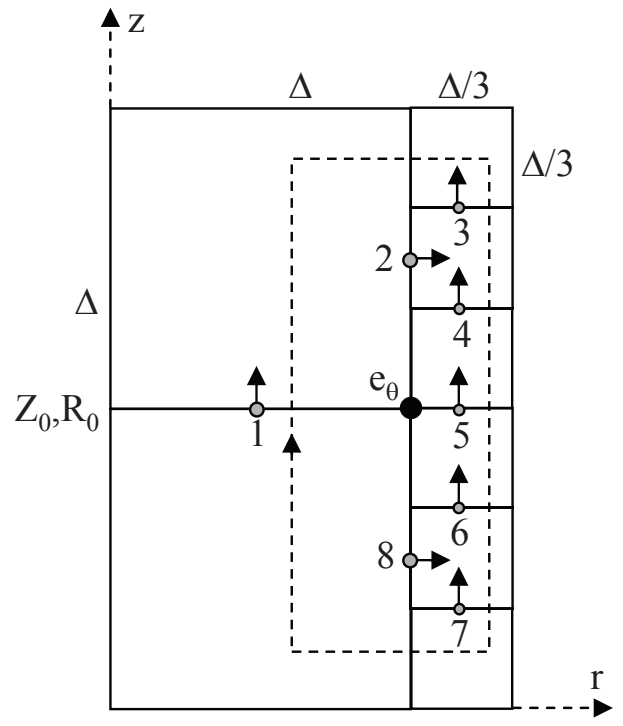

Figure 7: Updating of $\vec{E}_{\theta}$ at the subgrid edge $r=R_{0}$

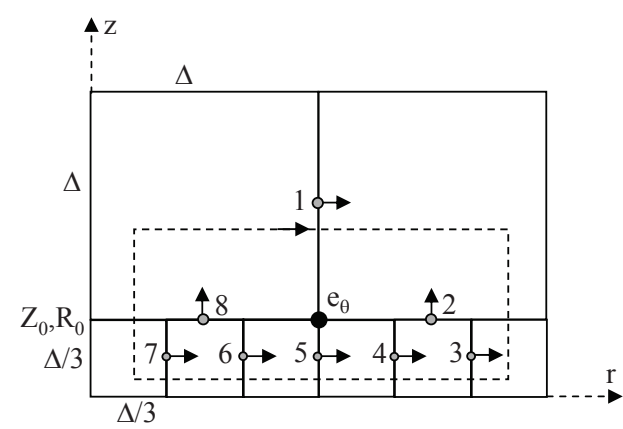

Figure 8: Updating of $\vec{E}_{\theta}$ at the subgrid edge $z=Z_{0}$

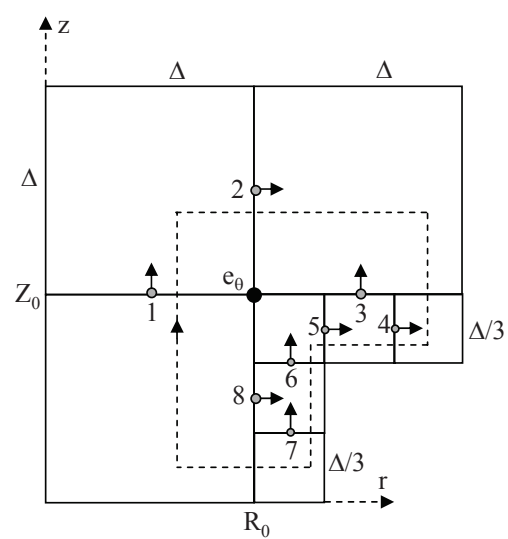

Figure 9: Updating of $\vec{E}_{\theta}$ at subgrid corner $r=R_{0}, z=Z_{0}$. 
[24] for a proof of the stability of this leapfrog scheme both in the absence and presence of subgridding, provided the time step $\Delta t$ respects the Courant limit of the size of the smallest grid, i.e. $c \Delta t \leq \Delta_{\text {finest }} / f(M)$, where $f(M)$ is roughly proportional to $M$ [1]. All eigenvalues of $\mathbf{A}$ are located on the unit circle.

Equations (35) and (36) suffice to write down an algorithm. However, starting from these equations it is elucidating to see how the proposed method takes care of the updating at subgridding boundaries. Let us start with $b_{r}$. Looking at Fig. 1 and supposing that $b_{r}$ is on a subgridding edge (say at $r=R_{0}$ ) and because after assembling the grid we only use coarse grid values, updating of $b_{r 1}$ requires the value of $e_{z 1}$ at the same place and the neighboring $e_{\theta, 00}$ and $e_{\theta, 10}$ values. To obtain the necessary value of $e_{\theta}$ at $z=Z_{0}+\Delta / 2$, (36) will enforce a linear interpolation, i.e. the $e_{\theta}$ value used at $z=Z_{0}+\Delta / 2$ will simply be $\frac{e_{\theta, 00}+e_{\theta, 10}}{2}$. This is of course what we would have expected right away. An analogous reasoning holds when updating $b_{z}$. For $b_{z 1}$ e.g. the value of $e_{r 1}$ at the same place and the neighboring $e_{\theta, 10}$ and $e_{\theta, 11}$ values are needed. To obtain the necessary value of $e_{\theta}$ at $r=R_{0}+\Delta / 2$, (36) now enforces an interpolation using the functions $f_{1}(r)$ and $f_{2}(r)$ defined in (2), i.e.

$$
e_{\theta, r=R_{0}+\Delta / 2}=f_{1}(r) e_{\theta, 10}+f_{2}(r) e_{\theta, 11}
$$

Coarse $b_{\theta}$ components always have coarse $e_{r}$ and $e_{z}$ neighbors and updating them is never problematic. Fine $b_{\theta}$ near the subgridding boundary are updated directly using the neighboring coarse $e_{r}$ and/or $e_{z}$.

Updating e-fields at subgridding boundaries turns out to be more complicated. This will be illustrated by the subgridding example of Fig. 3 studied above. We will use the numbering convention of Fig. 3b. To update $e_{r, 1}$ $\left(r=R_{0}+\Delta / 2, z=Z_{0}\right)$ we need the coarse value of $b_{z, 1}$ at the same point, the coarse grid value $b_{\theta, 5}$ at $r=R_{0}+\Delta / 2, z=Z_{0}+\Delta / 2$ and three fine $b_{\theta}$ values, i.e. $b_{\theta, 9}, b_{\theta, 12}$ and $b_{\theta, 15}$ all at $z=Z_{0}-\Delta / 6$ and resp. at $r=R_{0}+\Delta / 6$, $r=R_{0}+\Delta / 2$ and $r=R_{0}+5 \Delta / 6$. Eqn. (36) leads to the following weighted value of $e_{r, 1}$ :

$$
\begin{aligned}
& \epsilon \mu \frac{d e_{r, 1}}{d t}=\frac{-3}{2 \Delta} b_{\theta, 5}-M b_{z, 1}\left(\frac{1}{12} \frac{1}{R_{0}+\Delta / 6}+\frac{1}{12} \frac{1}{R_{0}+5 \Delta / 6}+\frac{10}{12} \frac{1}{R_{0}+3 \Delta / 6}\right) \\
& \quad+\left(\frac{1}{2 \Delta}-\frac{1}{6\left(R_{0}+\Delta / 2\right)}\right) b_{\theta, 9}+\left(\frac{1}{2 \Delta}\right) b_{\theta, 12}+\left(\frac{1}{2 \Delta}+\frac{1}{6\left(R_{0}+\Delta / 2\right)}\right) b_{\theta, 5}(39)
\end{aligned}
$$

We have again restricted ourselves to the simple case of a completely homogeneous medium with material parameters $\epsilon$ and $\mu$. An analogous reasoning 
holds when updating $e_{z}$. Suppose that we take a similar example as in Fig. 3 but that we now place the subgridding boundary at $r=R_{0}+\Delta$. Let us stick to the numbering of Fig. 3b by counterclockwise rotating the figure over 90 degrees. To update $e_{z, 1}\left(r=R_{0}+\Delta, z=Z_{0}+\Delta / 2\right)$ we now need the course value of $b_{r, 1}$ at the same point, the coarse grid value $b_{\theta, 5}$ at $r=R_{0}+\Delta / 2, z=Z_{0}+\Delta / 2$ and three fine $b_{\theta}$ values, i.e. $b_{\theta, 9}, b_{\theta, 12}$ and $b_{\theta, 15}$ all at $r=R_{0}+7 \Delta / 6$ and resp. at $z=Z_{0}+\Delta / 6, z=Z_{0}+\Delta / 2$ and $z=Z_{0}+5 \Delta / 6$. Eqn. (36) now shows that the corresponding weighted value of $e_{z, 1}$ is:

$$
\begin{aligned}
\epsilon \mu \frac{d e_{z, 1}}{d t} & =\frac{-3}{2 \Delta} \frac{R_{0}+\Delta / 2}{R_{0}+\Delta} b_{\theta, 5}-\frac{M}{R_{0}+\Delta} b_{r, 1} \\
& +\frac{1}{2 \Delta} \frac{R_{0}+\Delta(1+1 / 6)}{R_{0}+\Delta}\left(b_{\theta, 9}+b_{\theta, 12}+b_{\theta, 15}\right)
\end{aligned}
$$

The updating formula for $e_{z}$ is simpler than the one for $e_{r}$. This is a consequence of the fact that in the z-direction linear interpolation intervenes while in the r-direction a more complicated updating as in (38) plays a role: in cylindrical coordinates, "up" and "down" are equivalent, but "left" and "right" are not.

The updating strategy for $e_{\theta}$ is more complicated and depends on the exact position of the anchor point. Figs. 7, 8 and 9 shows the three possible situations. In Fig. 7, $e_{\theta}$ is located at an $r$ is constant edge, here at $r=R_{0}+\Delta$ with $z=Z_{0}+\Delta$. To update $e_{\theta}$ neighboring values of $b_{z}$ and $b_{r}$ are needed. From (36) one derives that

$\epsilon \mu \frac{d e_{\theta}}{d t}=\frac{3}{2 \Delta} b_{z, 1}+\frac{1}{\Delta}\left(b_{r, 2}-b_{r, 8}\right)-\frac{1}{6 \Delta}\left(b_{z, 3}+b_{z, 7}\right)-\frac{1}{3 \Delta}\left(b_{z, 4}+b_{z, 6}\right)-\frac{1}{2 \Delta} b_{z, 5}$

In points 1, 2 and 8 course grid values are used, in the other ones fine grid values. Fig. 8 shows the analogous case for updating $e_{\theta}$ at an $z$ is constant edge, here at $z=Z_{0}$ with $R=R_{0}+\Delta$. The update equation now becomes:

$$
\begin{aligned}
\epsilon \mu \frac{d e_{\theta}}{d t} & =\frac{3}{2 \Delta} b_{r, 1}+\left(b_{z, 2}-b_{z, 8}\right)\left(\frac{-1}{\Delta}+\frac{1}{6}\left(\frac{1}{6 R_{0}+11 \Delta}-\frac{1}{6 R_{0}+7 \Delta}\right)\right) \\
& -\frac{1}{6 \Delta}\left(e_{r, 3}+e_{r, 7}\right)-\frac{1}{3 \Delta}\left(b_{r, 4}+b_{r, 6}\right)-\frac{1}{2 \Delta} b_{r, 5}
\end{aligned}
$$

Here again, in points 1, 2 and 8 course grid values are used, in the other ones fine grid values. The most intricate situation arises when $e_{\theta}$ is located at a 
corner point as shown in Fig. 9 for $z=Z_{0}$ and $R=R_{0}$. In this case we have that

$$
\begin{aligned}
\epsilon \mu \frac{d e_{\theta}}{d t} & =\frac{9}{8 \Delta}\left(b_{z, 1}+b_{r, 2}\right)+\left(\frac{-3}{4 \Delta}+\frac{1}{8\left(6 R_{0}+5 \Delta\right)}-\frac{1}{8\left(6 R_{0}+\Delta\right)}\right) b_{z, 3} \\
& -\frac{1}{8 \Delta}\left(b_{r, 4}+b_{z, 7}\right)-\frac{1}{4 \Delta}\left(b_{r, 5}+b_{z, 6}\right)-\frac{3}{4 \Delta} b_{r, 8}
\end{aligned}
$$

Coarse grid point are at 1, 2, 3 and 8, fine ones at 4, 5,6 and 7. By the dashed lines in Figs. 7, 8 and 9 we have indicated the way in which the anchor points are ordered when calculating the corresponding time derivative of the $e_{\theta}$ field component. This reminds one of Ampère's law but one should be careful when wanting to interpret the obtained formula's in this way. In the absence of subgridding the restriction operators $A_{E}$ and $A_{B}$ only have 1 and 0 entries. In the subgridding case however these operators and also $\left[\star_{\epsilon}\right]$ and $\left[\star_{\mu}^{-1}\right]$ will depend on the explicit form of the curl-operator (8) and hence on the curl inclusion properties of the basis functions. Consequently, the proposed method imposes a strong relationship between the time-domain updating equations at the subgridding boundaries and the specific form of the curl operator.

\section{Numerical results}

First, stability, grid reflection and grid dispersion are investigated. Next the accuracy of the method is illustrated by determining the resonance frequencies of a toroidal cavity. This is followed by the study of a reentrant cylindrical particle accelerator cavity, showing that subgridding can substantially reduce CPU-time and memory requirements while retaining accuracy. Further research will focus on plasma applications, the results of which will be presented elsewhere.

\subsection{Stability}

In what follows we consider toroidal cavities (except for the cylindrical cavity example of Section 7.5) with PEC boundaries and focus on a particular angular mode with index $M$ implying a $\cos (M \theta)$ dependence for $e_{r}, e_{z}$ and $b_{\theta}$ and $\sin (M \theta)$ dependence for $e_{\theta}, b_{r}$ and $b_{z}$.

In a first example we explicitly calculated the 349 eigenvalues of the amplification matrix A for the very simple configuration of Fig. 10 and for $M=27$. The problem space counts $5 \times 5$ course grid cells of $1 \mathrm{~cm}$ by $1 \mathrm{~cm}$, 


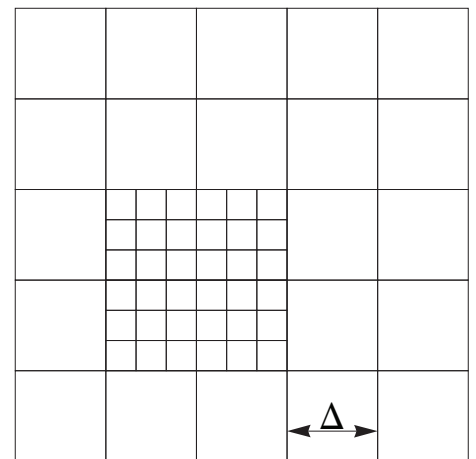

Figure 10: A simple configuration for the exact eigenspectrum calculation.
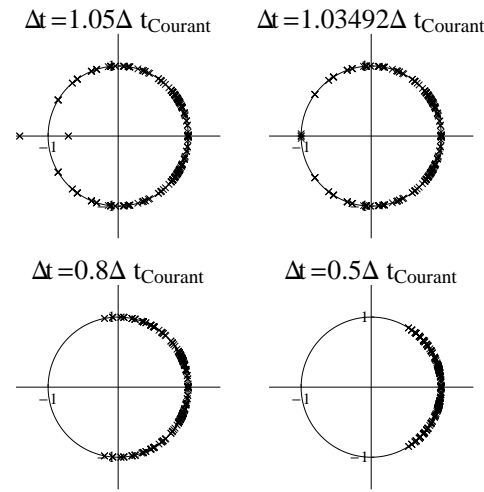

$\Delta \mathrm{t}=0.5 \Delta \mathrm{t}_{\text {Courant }}$
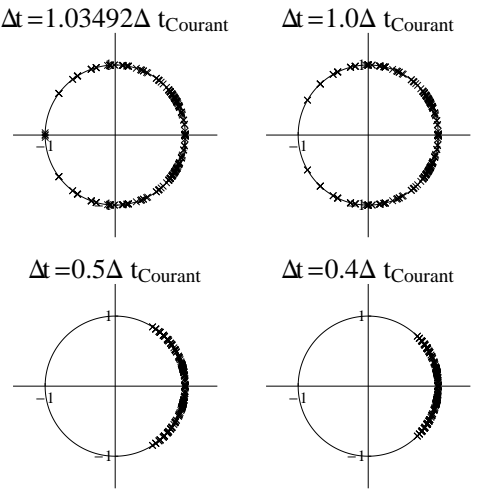

Figure 11: The eigenspectrum of the discrete amplification matrix for the simple configuration of Fig. 10 and for various values of $\Delta t$.

four of which have been subdivided in 9 fine grid cells. The cavity is filled with air, $R_{0}=2 \mathrm{~m}$ and $z_{0}=0 \mathrm{~m}$ (but the results remain completely invariant under a change of $z_{0}$ ). The behavior of the eigenvalues versus $\Delta t$ is shown in Fig. 11. For a suitable choice of $\Delta t$ obeying the Courant condition $\left(\Delta t=\Delta_{\text {Courant }}\right)$ for the smallest cells, i.e. $c \Delta t=\frac{\Delta}{3 \sqrt{2}} \approx 0.236 \Delta$, all eigenvalues are on the unit circle. as shown in Fig. 11. For a smaller time step, these eigenvalues move on the unit circle towards point $(1,0)$ as in Fig. 11 for $c \Delta t=0.8,0.5$ and $0.4 \Delta_{\text {Courant }}$. However, one remarks that when increasing $\Delta t$ slightly beyond the Courant limit, up to $c \Delta t=1.03492 \Delta_{\text {Courant }}$, stability is still preserved. We again refer to [24] for a thorough discussion, proving that the mass lumping in most cases results in an improved stability. When further increasing $\Delta t$, as shown in Fig. 11 for $c \Delta t=1.05 \Delta_{\text {Courant }}$, a negative real eigenvalue outside the unit circle is found and the time stepping will no longer remain stable. Note that in classical BOR-FDTD [1], the time step must be chosen roughly inversely proportional to the angular mode number. Since we do not consider cells close to the $r=0$ axis, we can use a time-step much larger than in BOR-FDTD while still preserving stability. As remarked by one of the reviewers, having all eigenvalues on the unit circle is a necessary but not a sufficient condition for stability. The eigenvalues on the unit circle must also be simple or nondefective [26].

Although the eigenspectrum results above indeed confirm the theoretical underpinning of the theory given in [17], asserting that the subgridding methodology we used automatically leads to a stable algorithm, we further assessed 


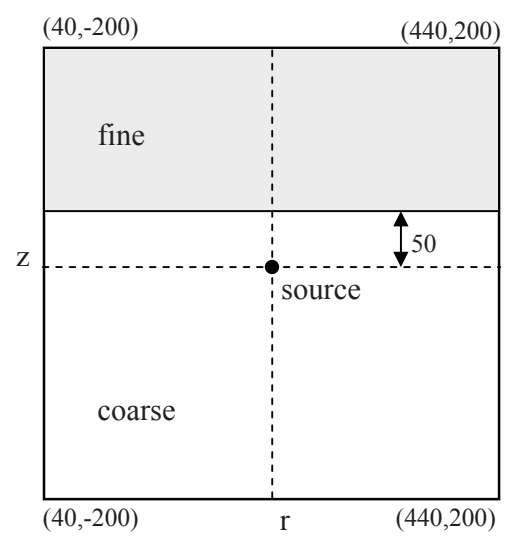

Figure 12: Simulation domain for grid reflection and grid dispersion calculations.

the long term stability in the examples given below in Section 7.4, by letting the algorithm run for a very large amount of time steps, i.e. several $10^{5}$ steps. No instability could be detected.

\subsection{Grid reflection}

Ideally, waves should propagate into the subgridded area without any reflection. To investigate the behavior of our technique, a sinusoidal line source with a $M=0$ (i.e. $\theta$-independent source) in the toroidal direction and with a wavelength $\lambda=N \Delta_{\text {coarse }}$ was used, with $N$ ranging between 6 and 100 . The cross-section of the considered configuration is that of Fig. 12. The simulation domain measures 200 by $200 \Delta_{\text {coarse }} \times \Delta_{\text {coarse }}$ cells with the left boundary located at $40 \Delta_{\text {coarse }}$ from the axis. The intersection point $S$ of the source loop with the $(\mathrm{r}, \mathrm{z})$-plane is located in the center of the simulation domain. In the lower part of the simulation domain the course discretization $\Delta_{\text {coarse }}$ is used, while the upper gray part is subgridded with $\Delta_{\text {fine }}=\frac{\Delta_{\text {coarse }}}{2}$. The boundary between the subdomains is located at $50 \Delta_{\text {coarse }}$ from the center. To assess the grid reflection, a first simulation is performed only using the course grid over the entire domain. The total simulation time is chosen such that the waves do not yet reach the outer boundaries. In the second simulation the coarse grid is used in the lower subdomain and the fine in the upper subdomain. The reflection coefficient plotted in Fig. 13 is defined as

$$
C_{r}=\frac{\operatorname{avg}\left(\left|\left(E_{\theta}\right)_{2}-\left(E_{\theta}\right)_{1}\right|\right)}{\operatorname{avg}\left(\left|\left(E_{\theta}\right)_{1}\right|\right.}
$$




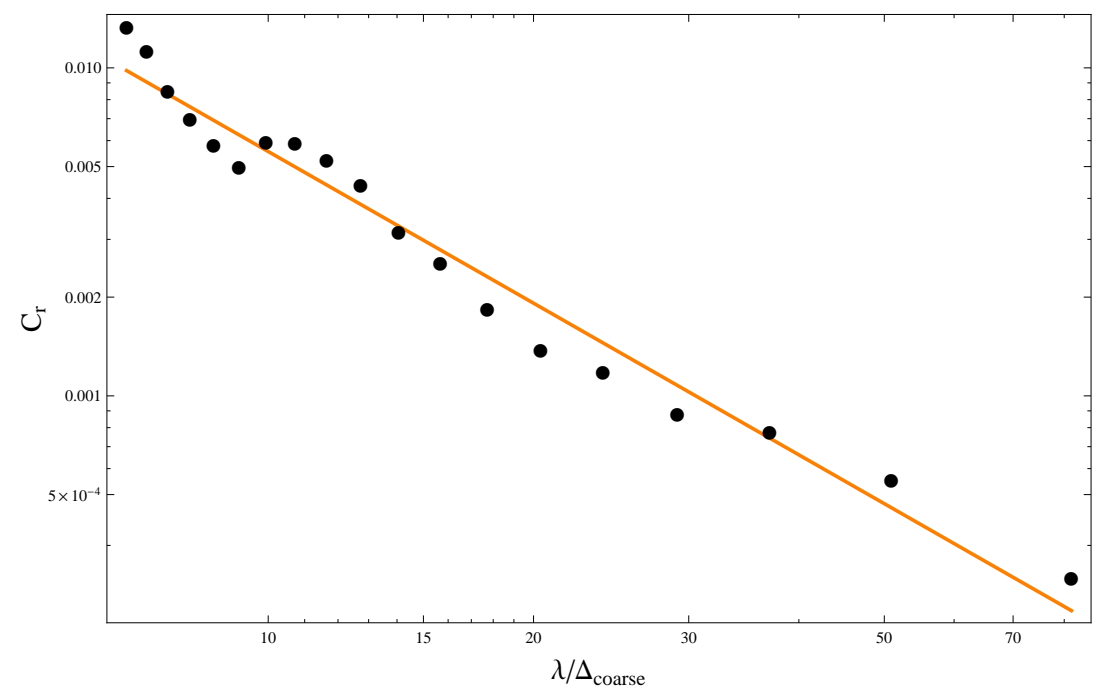

Figure 13: Grid reflection at the subgridding boundary

$C_{r}$ is the average absolute difference between the $\theta$-component of the electric field in the second and first simulation, divided by the corresponding value in the first simulation, whereby the average is taken over all cells of the lower, i.e. coarse cell, subdomain. This reflection coefficient decreases quickly as the amount of sampling points per wavelength increases, dropping below $6 \cdot 10^{-3}$ or $0.6 \%$ when $N \geq 10$. More important than the absolute value of $C_{r}$ is the fact that $C_{r}$ decreases as $\left(\frac{\lambda}{\Delta_{\text {coarse }}}\right)^{-1.5}$ which is the value predicted by Monk [27] for 2D cartesian FDTD.

\subsection{Grid dispersion}

Another phenomenon commonly encountered in FDTD simulations is the direction-dependent speed of light due to the regular discretization grid. To investigate this dispersion, we again start from the configuration of Fig. 12. At $t=0$ the current $i(t)$ in the loop (see also Fig. 14) is simultaneously switched on in all the points of the loop (corresponding to an $M=0$ mode). The current then starts oscillating sinusoidally with circular frequency $\omega$ (whereby due care is taken to smoothly switch the current on). We now determine all field values in the (z,r)-plane at a fixed instant $t_{0}$ and we chose $c t_{0}$ to be $170 \Delta_{\text {coarse }}$, with $c$ the speed of light in vacuum and $\Delta_{\text {coarse }}=\lambda / 7$. This implies that the wavefront in the $(\mathrm{r}, \mathrm{z})$-plane of Fig. 14 will reach no further 


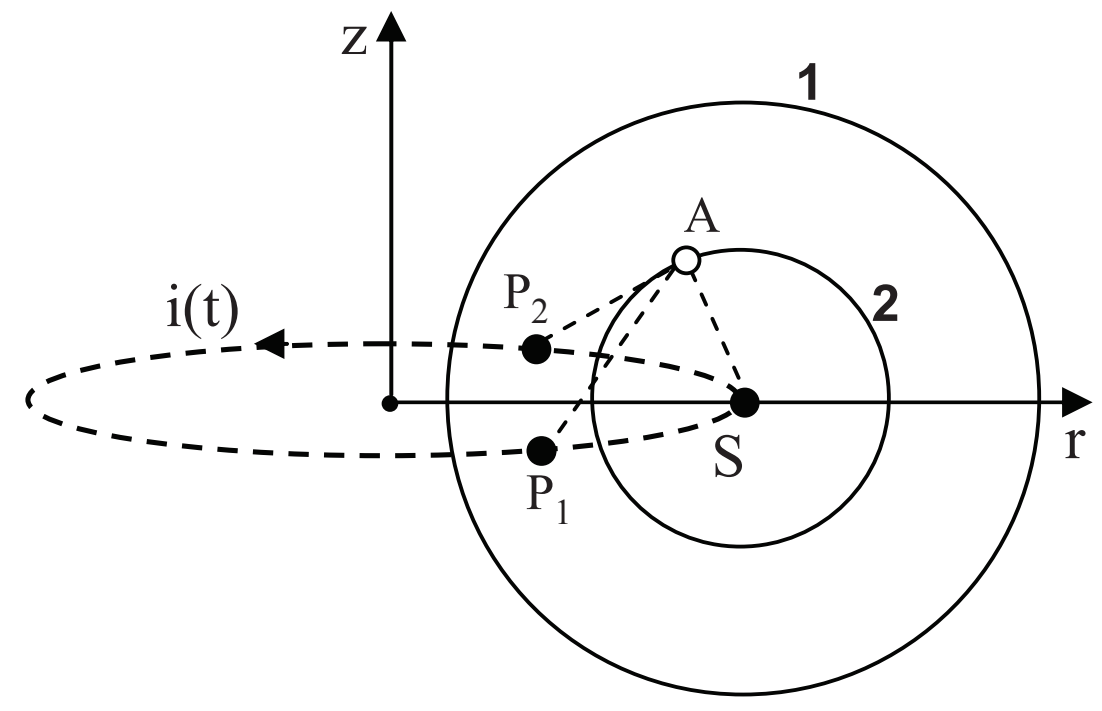

Figure 14: Current loop and field observation circles in the (r,z)-plane.

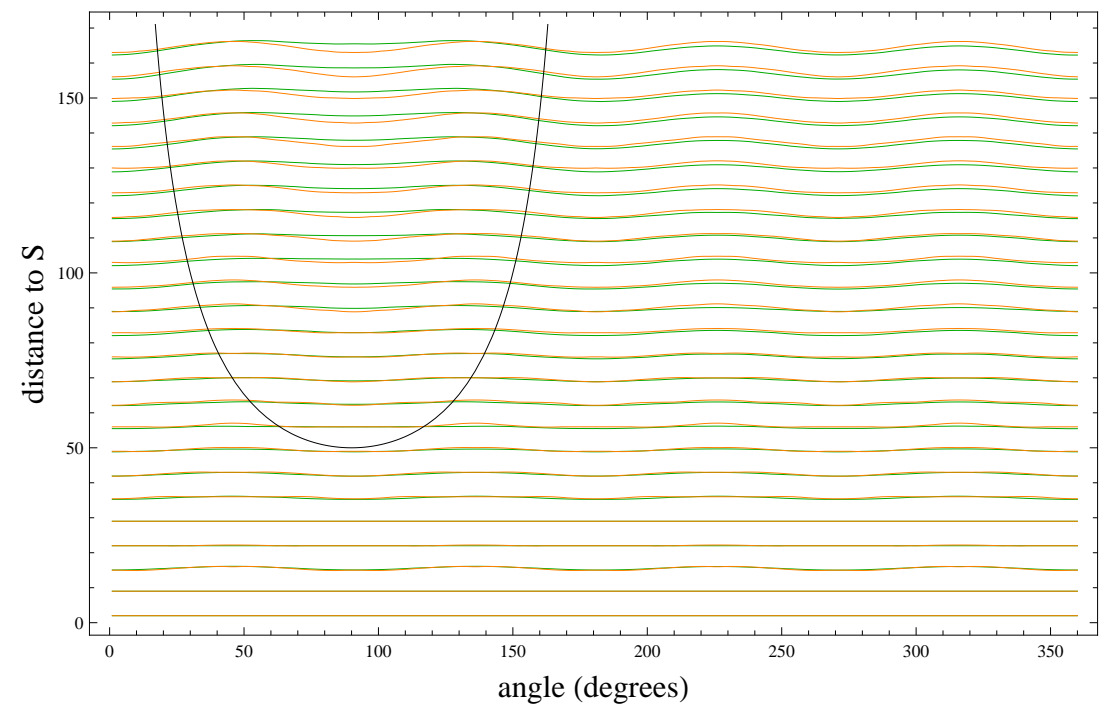

Figure 15: Distance between $S$ and wave maxima/ $\Delta_{\text {coarse }}$ vs. angle. Orange lines are without subgridding, green lines with subgridding. The black curve is the subgridding boundary. 
than the circle of radius $170 \Delta_{\text {coarse }}$ centered on $S$. This particular wavefront (denominated "1") originates from the point $S$ itself. Contributions from other points on the loop have to travel over a longer distance and cannot yet reach this outer wavefront. However, now consider another circular wavefront inside "1", such as the one denominated "2". Field contributions on this wavefront, e.g. in the point $A$, not only come from $S$ but also from other parts of the loop, e.g. from the points $P_{1}$ and $P_{2}$. The smaller the wavefront radius, the larger the portion of the loop points of which can contribute. Contributions to "2" coming from $S$ are obviously in phase. It is important to realize that contributions to " 2 " e.g. coming from points $P_{1}$ and $P_{2}$ on the loop will also be in phase with the contributions from $S$. Indeed, although the fields originating from $P_{1}$ and $P_{2}$ have to travel over a larger distance, the extra phase shift this involves is compensated by the fact that when the signal was emitted at $P_{1}$ and $P_{2}$, the phase of the source loop in these points lags behind as compared to the phase of $S$ at a later time. Or put differently: phase variations of the source points as a function of time and phase delays due to different path lengths compensate each other. The above reasonings lead to the following conclusion. When registering all fields at the fixed instant $t_{0}$, the fields will be in phase on circles such as " 1 " and "2" and indeed on any other circle centered on $S$. Consequently, field maxima (minima) will be found on some of these circles and such circles will be spaced by the wavelength. It must be emphasized that on such a "maximum" circle, the value of the maximum is not constant over the whole circle. Points on the circle closer to the center of the current loop will exhibit higher values than points further away. This is due to the 1 /distance decrease of source point contributions.

The phenomena described above crucially depend on the direction independent velocity of light. Hence, grid dispersion and the influence of subgridding can be clearly assessed in this way. For the parameters given above $\left(c t_{0}=170 \Delta_{\text {coarse }}\right.$ and $\left.\Delta_{\text {coarse }}=\lambda / 7\right)$ and for the non-subgridded case, the orange lines in Fig. 15 represent the numerically obtained loci of the maxima of the electric field. In order to give a clear picture of the results, we opted for a cartesian representation with the angle along the horizontal axis and with the distance to $S$, i.e the circle radius, along the vertical axis. Ideally all lines in Fig. 15 should be horizontal. It can be seen that with increasing distance to $S$, increasing dispersion errors become visible as an approximately $90^{\circ}$-periodic deviation from the ideal circles (i.e. horizontal lines in Fig. 15). This deviation is approximately $1.3 \%$ of the distance to $S$. One can also 


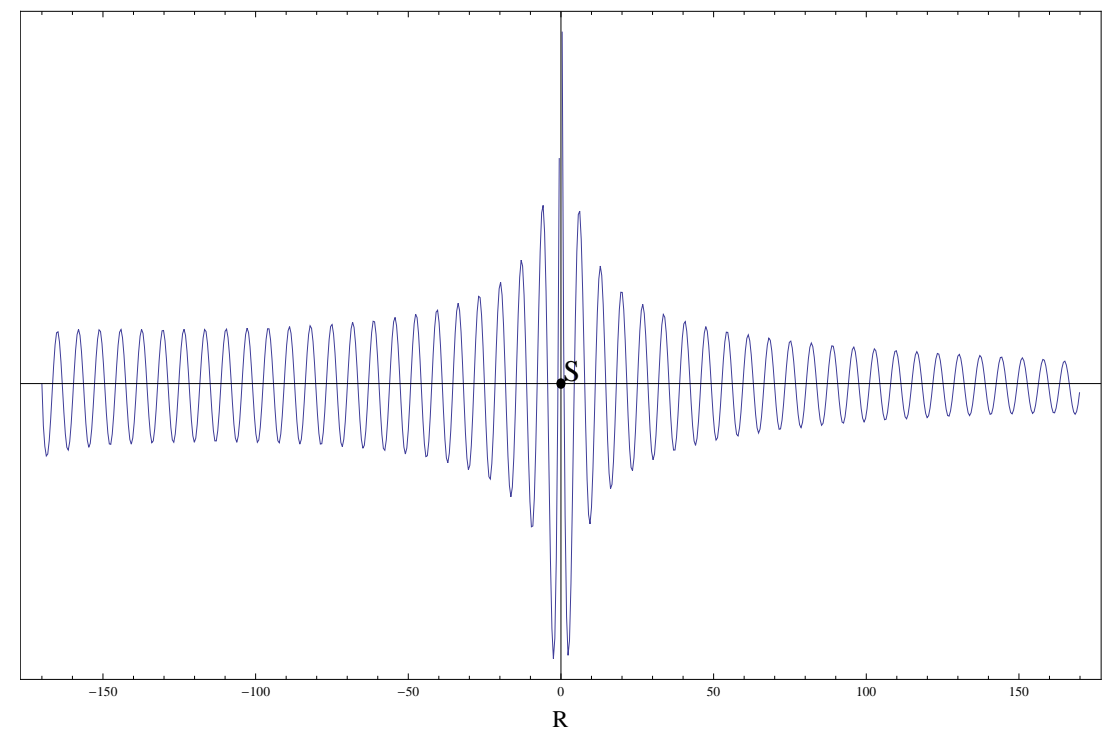

Figure 16: Electric field amplitude at $t_{0}$ along the r-axis.

clearly see that the maxima are spaced by $\lambda$. The dispersion error decreases as the amount of discretization points per wavelength increases. For 14 points per $\lambda$ we obtained a deviation of $0.4 \%$.

Next, the numerical experiment was repeated while introducing subgridding $\left(\Delta_{\text {fine }}=0.5 \Delta_{\text {coarse }}\right)$ in the gray part of Fig. 12 , corresponding to the area inside the black curve on Fig. 15. It is clear that the dispersion error decreases inside the subgridded area as the green lines are flatter than the orange ones and that little or no additional dispersion error is visible at the subgridding edge. Finally, to make clear that the value of the maximum varies along each circle, some additional data are given in Fig. 16. This figure shows the electric field amplitude along the r-axis $(z=0)$ starting in the leftmost point of circle " 1 " and ending in its rightmost point. Remarks that the position of the maxima (and minima) is symmetrical with respect to the midpoint $S$. $R=0$ corresponds to $S$ itself. For corresponding maxima, the absolute value is higher to the left $(R<0)$ of $S$ and the closer to $S$, the higher the value.

\subsection{Toroidal cavity examples}

As a next example consider a perfectly conducting torus with a square cross-section of inner radius $2 \mathrm{~m}$, outer radius $4 \mathrm{~m}$ and a height of $2 \mathrm{~m}$. As 
coarse discretization length we select $\Delta$ to be $1 \mathrm{~cm}$ and this coarse grid is combined with a fine grid with discretization length $\Delta / 3=1 / 3 \mathrm{~cm}$. This fine grid is an asymmetrically positioned square starting $60 \mathrm{~cm}$ from the left of the cavity (at $r=2.6 \mathrm{~m})$, and $60 \mathrm{~cm}$ from the bottom of cavity $(z=60 \mathrm{~cm})$ with side length $34 \mathrm{~cm}$. A line current in the toroidal direction at the center of the cavity, i.e. at a radius of $3 \mathrm{~m}$, is used as the excitation. In the time-domain this source terms takes the form

$$
j_{\theta}(t)=\cos \left(\omega_{0} t\right) e^{-\left(\frac{t-t_{0}}{a}\right)^{2}}
$$

with a Fourier spectrum proportional to

$$
J_{\theta}(\omega) \propto e^{-j \omega_{0} t_{0}} e^{-\frac{a^{2}}{4}\left(\omega-\omega_{0}\right)^{2}}+e^{j \omega_{0} t_{0}} e^{-\frac{a^{2}}{4}\left(\omega+\omega_{0}\right)^{2}}
$$

Choosing appropriate values for $a, t_{0}$ and $\omega_{0}$ enables us to only excite frequencies within a frequency range of interest, taking care that $t_{0}$ is large enough such that $j_{\theta}(t=0) \approx 0$. The time step $\Delta t=1 \cdot 10^{-11} \mathrm{~s}$ is chosen slightly below the Courant limit with respect to the fine discretization length. First, we excite a low-frequency $M=0$ mode in a vacuum-filled cavity using a line source of center frequency $\omega_{0}=600 \mathrm{MHz}$, with $t_{0}=0.1 \mu \mathrm{s}$ and $a=0.04 \mu \mathrm{s}$. From the Fourier transform of the simulation results in each point of the grid, it is easy to derive the resonance frequency that occurs. Both the subgridded and the non-subgridded simulations show a first resonance frequency at $\omega=672 \mathrm{MHz}$. The difference is below the frequency resolution. The analytical solution for the lowest-mode frequency is $\omega=660$ $\mathrm{MHz}$. The difference between the simulated results and the exact result is approximately $2 \%$.

To further investigate the influence of the subgridding, a more complicated problem is considered by filling the cavity for $r>3.5 \mathrm{~m}$ with a dielectric material with $\epsilon=9 \epsilon_{0}$. Furthermore, we use a toroidal mode $M=5$ and a higher-frequency source with a Gaussian spectrum centered at $\omega_{0}=3.3 \mathrm{GHz}$, with $t_{0}=0.08 \mu \mathrm{s}$ and $a=0.02 \mu \mathrm{s}$. The resulting spectrum is shown in Fig. 17 . There is very little difference between the location of the resonance frequency in the subgridded and the non-subgridded case. A typical plot after $10^{4}$ time steps of $\left|E_{\theta}\right|$ over the cross-section is shown in Fig. 18. The presence of the dielectric material is clearly visible. $\left|E_{\theta}\right|$ is nicely continuous everywhere and in particular at the subgridding edge. Furthermore, the subgridded area only barely disturbs the symmetry between the upper and lower halves of the configuration. 


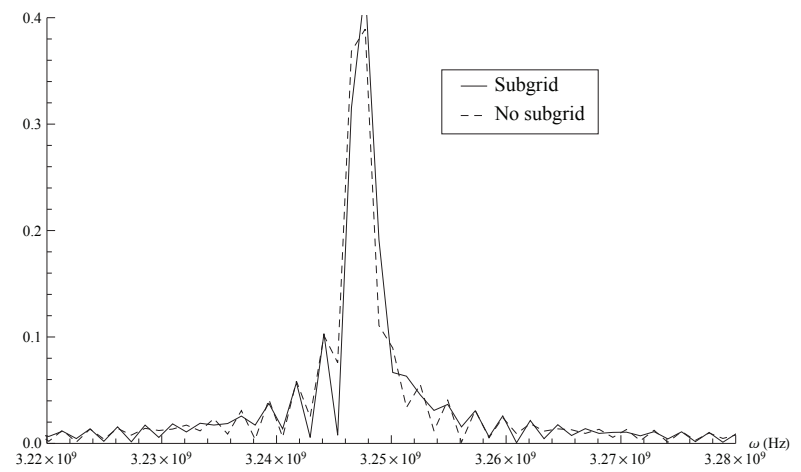

Figure 17: Comparison between a subgridded and a non-subgridded cavity for a toroidal $\mathrm{M}=5$ mode with a resonance frequency near $3.25 \mathrm{GHz}$

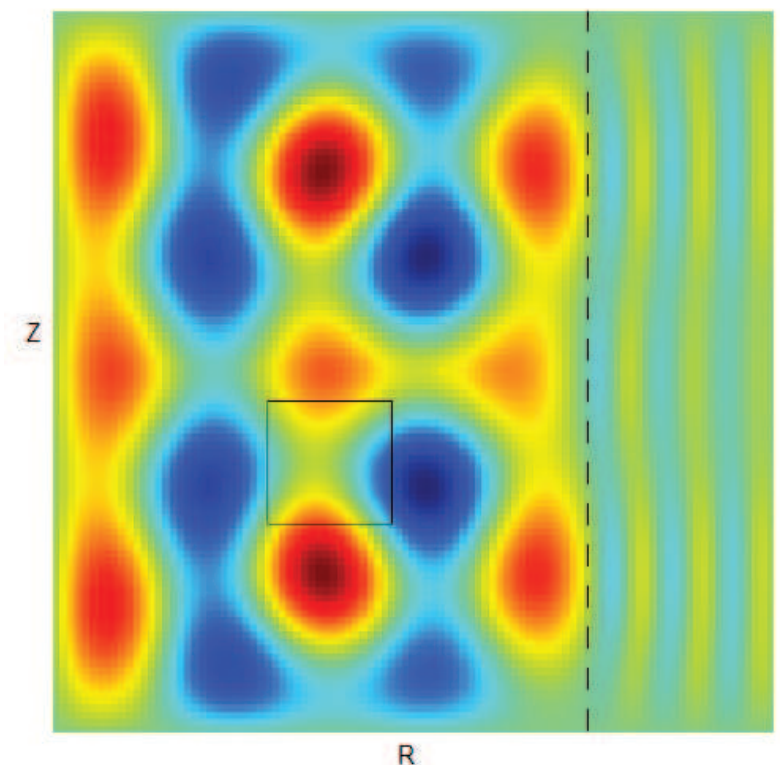

Figure 18: $\left|E_{\theta}\right|$ after $10^{4}$ steps. The rectangle indicates the boundary of the subgridded domain. 


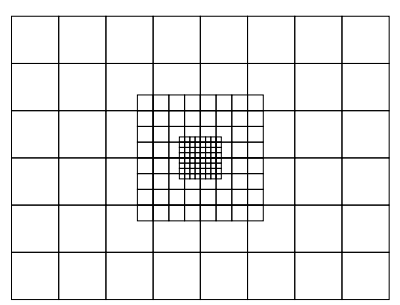

Figure 19: Multilevel subgridding

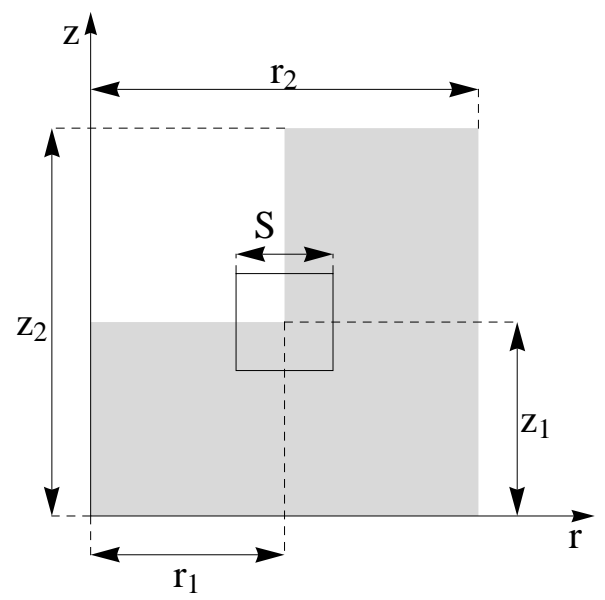

Figure 20: Reentrant cavity (gray) with sharp internal corner. The square of side $S=3$ $\mathrm{mm}$ is the $1 / 2$ subgridded part. $r_{1}=6.004 \mathrm{~mm}, r_{2}=42.29 \mathrm{~mm}, z_{1}=7.958 \mathrm{~mm}$, and $z=22.792 \mathrm{~mm}$.

In a last simulation, we examined multilevel subgridding: a part of the subgridded area is itself subgridded (Fig. 19). Compared with a non-subgridded simulation, the resonance frequencies shift by about $0.4 \%$. In all these examples, all simulations have run for at least $3 \cdot 10^{5}$ time steps and, as expected, no sign of instabilities has been observed.

\subsection{Reentrant cylindrical cavity}

In $[28,29]$, the problem of calculating the resonant frequencies of reentrant cylindrical cavities, such as those encountered in Alvarez-type particle accelerators, is discussed. Due to the presence of sharp internal corners at which the electric field becomes large and varies strongly over a small dis- 
tance, FDTD methods are hard to use for this kind of problem. Here, we will revisit the configuration of [28] (Fig. 20, Figs. 1 and 2 in [28]), first with a uniform fine discretisation, and then with subgridding near the sharp corner. In this way, we can obtain nearly the same result using only about $(1 / 2)^{2}=1 / 4$ of the memory needed for the fine discretization. Note that this is a cylindrical configuration, not a toroidal one, so some care must be taken to properly simulate the $r=0$ axis. The subgridding does not intersect with the $r=0$ axis, so our method reduces to classical BOR-FDTD there, allowing the use of the on-axis update equations described in [1]. At $M=0$, it suffices to introduce some minor changes to the mass lumped electric mass matrix $\left[\star_{\epsilon}\right]$ to make this method give the correct BOR-FDTD equations at $r=0$ as well.

The dimensions given in Fig. 20 are those of [28]. In a first simulation a uniform grid of size $\Delta=0.1 \mathrm{~mm}$ is used. After exciting the cavity using a time-domain source with a broad frequency spectrum, we fourier-transformed the result to find the lowest resonance peak at $2.1 \mathrm{GHz}$, as predicted in [28].

In a second simulation the overall discretization length was doubled to $\Delta=0.2 \mathrm{~mm}$, except for a small subgridding square of side $S=3 \mathrm{~mm}$ around the internal corner (Fig. 20), where the discretization length remains fine at $\Delta=0.1 \mathrm{~mm}$. By doing so, we decrease the memory used by the simulation by almost a factor 4 . The running time decreases as well because almost 4 times less unknowns have to be updated every time step. Nevertheless, we still have to obey the Courant limit of the small cells and thus have to run the simulation for the same amount of time steps. The resulting fields are barely distinguishable from those of the uniform fine simulation. Most importantly, the excited resonance peak is still at $2.1 \mathrm{GHz}$.

\section{Conclusions}

We have extended the provably stable subgridding method of [17] to BOR-FDTD. Using appropriate mass-lumping techniques yields an explicit and stable time-stepping algorithm provided the Courant limit of the fine mesh cells is respected (although the mass-lumping can lead to a relaxing of this limit). The FDTD updating equations at subgrid edges and corners, resulting from the mathematical machinery, cannot readily be interpreted intuitively. Some simple cases provide the reader with the necessary insight. Several numerical examples, using toroidal cavities, have demonstrated that the proposed subgridding is indeed stable and by considering the eigenmodes 
of such cavities it is also shown that the method remains very accurate even in the case of multilevel subgridding. We also provided some data on the subgridding reflection and disperion behavior. Finally, a short study of the resonant behavior of an accelerator type reentrant cylindrical cavity, shows that subgridding can lead to savings in CPU time and memory while retaining accuracy.

In the future, we intend to extend our method to be able to subgrid plasmas instead of simple dielectrics. In that case the current and the constitutive equation connecting the current to the fields need to be treated in a special way (using an auxiliary differential equation).

\section{References}

[1] A. Taflove, S. Hagness, Computational Electrodynamics: The FiniteDifference Time-Domain Method,3rd ed., Artech House, 2005.

[2] F. L. Teixeira, FDTD/FETD methods: a review on some recent advances and selected applications, Journal of Microwaves and Optoelectronics 6 (2007) 83-95.

[3] T. D. Tsiboukis, Synthesis Lectures on Computational Electromagnetics, Morgan \& Claypool Publ., 2006.

[4] D. N. Smithe, Finite-difference time-domain simulation of fusion plasmas at radiofrequency time scales, Physics of Plasmas 14 (2007) 25372549 .

[5] T. Stix, Waves in Plasmas, American Institute of Physics, 1992.

[6] I. I. T. B.J. Green, P. Teams, ITER: burning plasma physics experiment, Plasma Physics and Controlled Fusion 45 (2003) 687.

[7] E. R. Rebhan, G. V. Oost, Thermonuclear burn criteria for D-T plasmas, Fusion Science and Technology 49 (2006) 16-26.

[8] J. Y. J. Shibayama, B. Murakami, H. Nakano, LOD-BOR-FDTD algorithm for efficient analysis of circularly symmetric structures, IEEE Microwave and Wireless Components Letters 19 (2009) 56-58. 
[9] D. Davidson, R. Ziolkowski, Body-of-revolution finite-difference timedomain modeling of space-time focusing by a three-dimensional lens, J. Opt. Soc. Amer. A 11 (1994) 1471-1490.

[10] R. M. Y. Chen, P. Harms, Finite-difference time-domain algorithm for solving Maxwell's equations in rotationally symmetric geometries, IEEE Transactions on Microwave Theory and Techniques 44 (1996) 832-839.

[11] Y. Y. H.-L. Chen, B. Chen, D.-G. Fang, Unconditionally stable ADIBOR-FDTD algorithm for the analysis of rotationally symmetric geometries, IEEE Microwave and Wireless Components Letters 17 (2007) 304-306.

[12] J. Chen, J. Wang, A novel body-of-revolution finite-difference timedomain method with weakly conditional stability, IEEE Microwave and Wireless Components Letters 18 (2008) 377-379.

[13] P. Thoma, T. Weiland, A consistent subgridding scheme for the Finite Difference Time Domain method, Int. J. Num. Model.: Electr. Netw., Devices and Fields, 9 (1996) 359-374.

[14] E. O. M. Okoniewski, M. Stuchly, Three-dimensional subgridding algorithm for FDTD, IEEE Transactions on Antennas and Propagation 45 (1997) 422-429.

[15] S. Wang, F. L. Teixeira, R. Lee, J.-F. Lee, Optimization of subgridding schemes for FDTD, IEEE Microwave and Wireless Component Letters 12 (2002) 223-225.

[16] B. Donderici, F. L. Teixeira, Improved FDTD subgridding algorithms via digital filtering and domain overriding, IEEE Transactions on Antennas and Propagation 53 (2005) 2938-2951.

[17] R. A. Chilton, H-, P- and T-refinement strategies for the finitedifference-time-domain (FDTD) method developed via finite-element (FE) principles, Ph.D. thesis, Ohio State University, 2008.

[18] L. Kulas, M. Mrozowski, Low-reflection subgridding, IEEE Transactions on Microwave Theory and Techniques 53 (2005) 1587-1592. 
[19] D. P. K. Xiao, J. Drewniak, A three-dimensional FDTD subgridding algorithm with separated temporal and spatial interfaces and related stability analysis, IEEE Transactions on Antennas and Propagation 55 (2007) 1981-1990.

[20] J. Bérenger, A Huygens subgridding for the FDTD method, IEEE Transactions on Antennas and Propagation 54 (2006) 3797-3804.

[21] M. F. Wong, V. F. Hanna, J. Wiart, Finite element formulation of spacetime subgridding scheme in the FDTD method, Int. J. Num. Model 13 (2000) 127-137.

[22] M. Bonilla, M.-F.Wong, V. F. Hanna, A finite-element formulation for FDTD subgridding, Microw. Opt. Technol. Lett. 32 (2002) 104-108.

[23] B. Donderici, F. L. Teixeira, Domain-overriding and digital filtering for 3D FDTD subgridded simulations, IEEE Microwave and Wireless Components Letters 16 (2006) 10-12.

[24] R. A. Chilton, R. Lee, Conservative and provably stable FDTD subgridding, IEEE Transactions on Antennas and Propagation 55 (2007) 2537-2549.

[25] R. Lee, A note on mass lumping in the finite element time domain method, IEEE Transactions on Antennas and Propagation 54 (2006) 760-760.

[26] S. Wang, F. L. Teixeira, Some remarks on the stability of time-domain electromagnetic simulations, IEEE Transactions on Antennas and Propagation 52 (2004) 895-898.

[27] P. Monk, Subgridding FDTD schemes, Applied Computational Electromagnetics Journal 11 (1996) 37-46.

[28] R. C. Carter, J. Feng, U. Becker, Calculation of the properties of reentrant cylindrical cavity resonators, IEEE Transactions on Microwave Theory and Techniques 55 (2007).

[29] R. Taylor, Calculation of resonant frequencies of re-entrant cylindrical electromagnetic cavities, Journal of Nuclear Energy, part C : Plasma Physics 3 (1961) 129-134. 